\title{
Agent Based Cucumber Plant Climbing Characteristic Simulation
}

\author{
Jing $\mathrm{Xu}^{1}$, Teng Miao ${ }^{1}$, Kai Song ${ }^{1,2^{*}}$, Lin Gao ${ }^{1}$ and Ying $\mathrm{Cui}^{1}$ \\ ${ }^{1}$ The College of Information and Electric Engineering, Shenyang Agricultural University, Shenyang, China \\ ${ }^{2}$ The College of Information and Engineering, Shenyang Ligong University Shenyang, China \\ ${ }^{*}$ Corresponding author
}

\begin{abstract}
This paper presents a method to simulate the plant behavioral characteristics based on Agent. The basic structure of plant Agent is introduced and the perception, behavior decisionmaking ability, motion ability of the plant Agent are given. Based on the plant Agent, climbing growth characteristics of cucumber is simulated. In this simulation, light factor, gravity factor, tendrils factor and human factor are considered, and a liner weighted method is used for behavior decision-making algorithm. Experiments show that the plant Agent can simulate plant behavioral characteristics realistically and generate simulation animation quickly.
\end{abstract}

Keywords-plant behavioral characteristics; agent; climbing growth

\section{INTRODUCTION}

In recent years, with the deepening of digital agriculture research and practice, research on the digital plant theory and technology system cause people more and more attention[1], in which plant behavior characteristics simulation is one of the important research problems of digital plant.

Agent is a hot research topic in the field of artificial intelligence. Due to the different areas of research, the concept of intelligent body has a different understanding , from the point of biological group modeling domains, agent is defined as a physical target , behavior and knowledge of the field, it can act on its own and the environment, and respond to the environment , with autonomy, communication , responsiveness, initiative, reasoning, collaboration and other basic characteristic attributes .In recent years, the modeling method based on agent has made great progress in the research on the behavior of biological community, and gradually formed a new method for generating computer animation. Such as Tu XiaoYuan [2], etc. developed artificial fish technology, Improve system [3] at New York University and Badler, etc. build virtual man JACK [4].The agent technology has been applied to the research of virtual plants. Such as Hanan etc. established plant diseases and pests - interaction model [5] and Benes etc. established plant community ecology system simulation model [6]. Some domestic scholars have started to work on the Agent of plants, but it is limited to the theoretical discussion, and it is not related to the specific implementation techniques [7-8].

This paper Uses agent technology, simulate the characteristics of cucumber plant climbing in greenhouse, proposes a method of cucumber climbing in the process of perception, decision-making and climbing movement, used to generate climbing characteristics simulation animation.

\section{Plant Agent Modeling}

Movement behavior in plants is a complex self-organizing and integrated process of interaction with the environment, their perception by the external environment changes, constantly adjust its nutrient allocation strategy and morphological structure, in order to achieve optimal growth state in the current environment. Plant agent can be divided into three parts, the perception system, the behavior decision system and the motion system. Perception system accepts the dynamic changes of the external environment, such as sunlight, exploring the space. Behavioral decision processing system is the core of plant intelligence, it is based on the professional knowledge base, the information of the outside world is classified processed, and the reaction mechanism of the plant characteristics is simulated through a certain decision algorithm. Growth, according to decision-making information, calculate motion parameters and structure parameters, and adjust the organ morphology and color texture. The structure of plant agent is shown in Figure I.

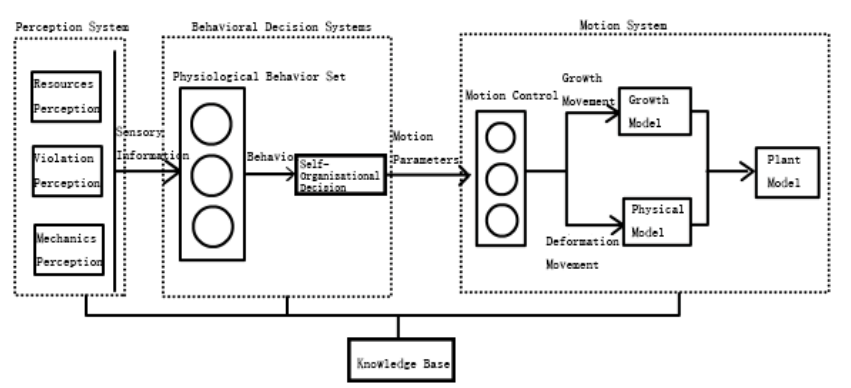

FIGURE I. STRUCTURE FIGURE OF PLANT AGENT

\section{A. Perception System}

In the characteristics of plants, the feeling is very important, plants have to perceive the surrounding environment in order to obtain energy, and is responsible for the perception of the task is the organs of plant body, such as the roots of plants can sense the underground water and fertilizer, photosynthetic organs of plants (such as leaves) can sense light distribution, the stems of plants can sense the size of the space. This paper adopts the direct questions environment model combined with computer graphics 
algorithm, to construct plant perceptual system so that plants can collect information in a virtual environment to support its life activities required. We divide plant sensing system into three subsystems: resource perception system, invasion perception system, force perception system.

1) Resource perception system: The plant will feel the position and distribution of the resources space, light, water, fertilizer etc. In order to simplify the modeling process, in the plant agent, assuming that the aboveground part of organs (leaves, buds, etc.) only induce light, the underground part organ (root) only induce the water resources. For the light sensor, the plant agents calculate the size of light which plant received. Lighting calculation is a complex issue. The total amount of light is the sum of direct light and scattered light, but in order to facilitate the calculation and the effect of direct light is greater, the effect of scattering light is not taken into account in the simulation. Meanwhile, the simulated light distributions have different methods, the easiest way is about to consider a point source light (namely the light is only from a point), More complex light source can be seen as a surface light source, we use the latter light simulation. For illumination within a scene, obey a hemisphere uniform distribution, as shown in (dashed line is light ray),Suppose light emitted from each point in the sky contains same light degree and ignore the effects of attenuation with distance, for the amount of light the plants at any position A is calculated by the following steps: In the first sampling uniform light point in hemisphere, the number of sampling points is $\mathbf{M}$; Secondly, for each light source point A, to determine whether to light A is blocked by other objects. Number of light points are located occluded is $\mathrm{N}$, then the amount of light received at point A is $\mathrm{M}-\mathrm{N}$.

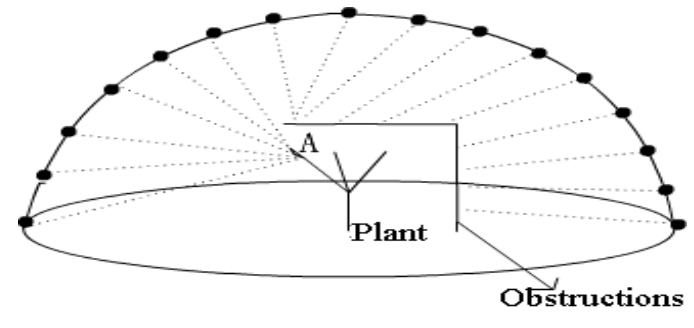

FIGURE II. LIGHT FLUX COMPUTING

For water, fertilizer induced mainly from plant roots organs Agent, The absorption of water, carbon and nitrogen is a more complicated mechanism, and many scholars have studied it from the point of view of the physiological model. This article from the perspective of simulation to simple the process as to the amount of each ingredient absorption, and the absorbed dose is simplified as proportional to the volume of the root system model, formula is as follows: $\mathrm{T}=\mathrm{V}^{*} \mathrm{k}$, among that $\mathrm{T}$ is the uptake, $\mathrm{V}$ is the root volume, $\mathrm{k}$ is the absorption factor (represented with the ability to absorb resources).

a) Force sensing system: The plants themselves can sense the external force, reflecting the different morphological changes, the study of the mechanical properties of plants is a difficult problem, so far the research involved is less. At present, many plants will be simplified as elastomers, elastic physics to simulate the mechanical properties of plants. For the power of perception, in plant agent only can accept the system of the incoming mechanical parameters, Such as when the system sets the gravity, the plant agent will perceive gravity.Deletion: Delete the author and affiliation lines for the second affiliation.

b) Invasion sensing system: Plants under the influence of biotic and abiotic factors, may occur a series of morphological, physiological and pathological changes in biochemical, hindering the normal growth and development process, called disease. Plant of organisms that cause diseases such as insects, fungi) will have a certain reaction, in the plant agent use against the perception of the perception, similar to the force sensing system, against the perception is passed through the system parameters information received.Selection: Highlight all author and affiliation lines.

\section{B. Behavioral Decision System}

Behavioral decision making system simulates the process of self-organizing of plants, and after receiving the information from the sensor, through internal algorithm, to calculate the current motion control parameters. For sensory information received, the plant will produce different physiological response, it is a complex nonlinear process, for the characteristics of different simulation or difference method may be used for structure method. This paper adopts a kind of behavior choice to simulate, for each aware message, will correspond to one or more of the following behavior and control parameters, and then through a series of priority selection and weighting process parameters, and ultimately determine the type of behavior and execution order and a series of parameter values.

\section{Motion Control System}

For the change movement, the control system is constructed based on the growth model, and the bud is the basic growth unit, each bud has apical meristem divide, copying and other activities, rely on internal factors(such as biological age, the number of separatist group, etc.) and external environmental factors (such as light, moisture, etc.) affect the activity of buds, During growth, buds may die, sleep, or generate flowering stems, which generates a stem to promote the growth and branching of plant plants, is the most important physiological functions. The shoots were divided into apical bud, lateral bud, apical buds located in the top of trunk or branches, lateral bud many born in axilla of leaves, The generating process of the bud can be expressed by the graph, as shown in Figure III. Initially, the new leaves start to grow on the side of the terminal bud, while the emergence of a new lateral buds in the leaf axils portion after bud will continue to grow and produce new stems, branches was in this differentiation process evolving. 

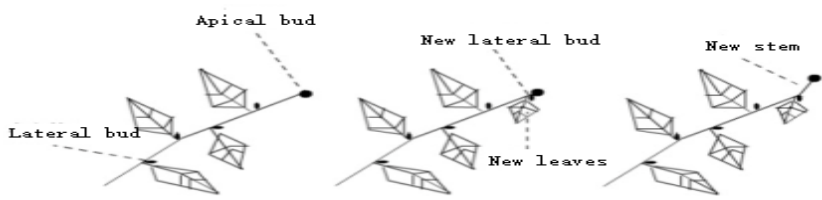

FIGURE III. THE GROWTH MOVEMENT

For the deformation motion, the control system is a set of multilevel physical system from the whole plant scale to the organ scale, in which the physical system is constructed by the method of land $[9,10]$.

\section{Organ Module}

Using contour and axis technology to construct the cucumber organ model, this method can through interactive quickly construct the morphology of major organs and can according to the dynamic control of organ morphology change, suitable for animation generation. Figure IV a variety of organ models generated by this method.

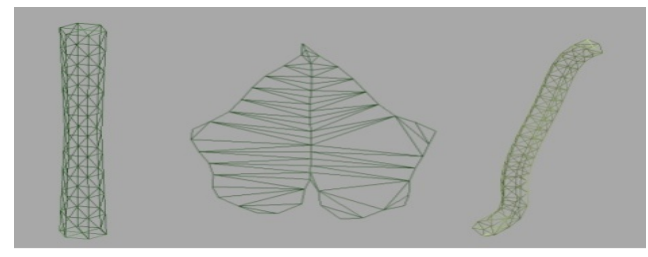

FIGURE IV. 3D MODEL OF PLANT ORGAN

\section{CUCUMBER Climbing CHARACTERISTICS Simulation}

Cucumber climbing is accompanied by the growth of the important characteristics, combining with the plant simulation technology for this feature in this paper. In the process of climbing, cucumber can sense light, water and fertilize, space and other environment information, but a greater impact on climbing mainly for the following acts: light, tendril space exploration, gravity and artificial tendril around. Cucumber climbing movement is a combination of changes and motions deformation process, the change movement plays a main role, so this article assumes that climbing behavior is a movement to change.

\section{A. Climbing Perception}

1) Light perception: The organ of bud is to produce stem, which determines the direction of climbing plants. In the process of bud producing sub-stem will be affected by light, try to grow to sufficient sunlight direction. Using the method in 2.1.3 to calculate the direction of maximum light intensity, if more than one direction, you can use the average method or a random selection method to obtain the final illumination direction, that direction is to achieve maximum light bud growth direction.

2) Tendrils space exploration: Cucumber tendril is the variation of lateral branch, was born in lateral bud, assuming that crown nearest tendrils will affect the direction of the new stem with, so just on the adjacent terminal bud tendrils space exploration can be. Tendrils borne position is the position of lateral buds, tendrils in plant health conditions, more grow obliquely about 45 degrees, in order to determine the growth direction tendrils. Tendril can only be carried out within a certain scope of exploration, the maximum range is called perception. Will the sites as a spatial point, that point as the origin, tendril growth direction as the center axis, perception scope for a certain distance $d$ from the origin, a collection of space a for a certain space Angle apart from the central axis, as shown in figure. Within the scope of the perception, randomly selected from a certain number of points as the tendrils of detection points, these points with the origin are connected to the origin to constitute a detection line, through the collision detection technology to judge whether to detect line segments intersect with the support scenario, if they have intersection, tendrils catch objects in this direction. When performing collision detection, firstly computing the intersection of the line with the plane which contains the triangle, followed by calculation of the coordinates of the intersection pointed, and to judge whether it is located in the triangle, and if so, then the line segment intersects with the triangle. In order to accelerate collision detection using octree technical cut up The static support model of the whole scene in advance, supplemented by AABB technology for pre-detection.

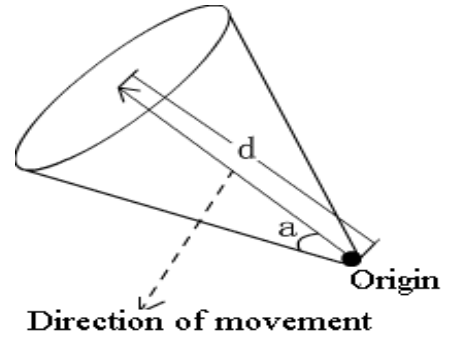

FIGURE V. THE STRATEGY OF TENDRILS EXPLORATION

3) Gravity sensing: Cucumber in the case of climbing will always subjected to the action of gravity, if supported by the support, the whole plant will fall to the ground, eventually climbing to the ground to grow. We set in our system agent affected by gravity, the new stem in the process of growth will be affected by the gravity of the direction vertical to the ground.

4) Artificial around the tendril perception: Artificial tendril around make cucumber would have been better around the support climbing upwards. Around the trailing plants in fact can be seen as an agent for Rio's perception of the phenomenon, the artificial tensile strength makes plant closer to the support direction, and guarantee the plant eventually grow up, so the artificial around tendril aware are approximated to two tension sensing in pairs, as shown in Figure VI, F1 is the upward pull, F2 to support the direction of the wire tension. 


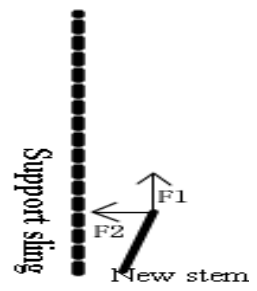

FIGURE VI. THE FORCE OF TWINING THE VINE BY HAND

\section{B. Climbing Behavioral Decision}

This article adopts the way of behavior synthesis as plant behavior decision-making method. For different perceptual information, the growth of new stems have different choice: Such as the perception of light, the new stems will choose to accept the direction of the largest amount of light as the growth direction, the spatial perception tendrils, the new stem will affected by a tensile force which towards tendril direction. For behavior under different perception, we use linear acceleration to determine the final growth direction of new stem, such as formula.

$$
\left\{\begin{array}{l}
\mathbf{D}=a \mathbf{S}+b \mathbf{T}+c \mathbf{G}+d \mathbf{F} \\
a+b+c+d=1
\end{array}\right.
$$

where in, $\mathrm{D}$ is the final growth direction, $\mathrm{S}$ is able to accept the maximum illumination unit direction vector, $\mathrm{T}$ is tendrils direction of pull, $\mathrm{G}$ is the gravitational direction, $\mathrm{F}$ is about the spread of the direction of pull, a, b, c, d are weighting factors, by setting different values can be simulated climbing conditions under different circumstances.

\section{Climbing Movement}

After get the growth direction, just using the change movement of the control system, the motion parameters is calculated according to the growth model, such as growth rate, the new stem length, width and other values.

\section{RESUlTS AND DisCUSSION}

The system implementation in VC2005 and OpenGL graphics engine programming environment, and the configuration of 1.96 GHZ CPU, DDR 2GB of memory and ATI RHD2400 graphics PC to experiment, to facilitate observation climbing characteristics of cucumber, show only the three-dimensional model of cucumber stem. Figure VII for cucumber artificial around tendril climbing system simulation animation effects in this paper, the weighting factor(a, b, c, d) is set to $0,0,0.2,0.8$ respectively, this group of parameters under the condition of artificial tendril around, the climbing plants are greatly influenced by artificial factors. Figure VIII by tendril climb simulation, the red line is the direction of tendril growth, weighting factor is set to $0,0.6,0.4$, 0.Figure VIII(a) in the middle of the plant due to the support tendrils are not looking, and the influence of gravity, circumferentially growth; and Figure VIII(b) plants rely on tendrils down the rope to climb upwards. Figure IX for the effect of combining two climbing behaviors, the early stage of the cucumber under the artificial management around the tendril growth (weighting factor is set to $0.0,0,0.2,0.2)$, after more than wire height, cancel the manual administration (weighting factor is set to 0.3 , $0.5,0.2,0$ ), cucumber to support through tendrils winding wire, attached to the wire on the growth, until tendrils couldn't come into contact with the wire, affected by gravity down growth, in order to display the sun's influence on plant climbing, scene were added occlusion cuboid (rectangular parallelepiped shown in red), since there is less directional light is blocked, so that the plants selected the opposite direction to climb. Figure $\mathrm{X}$ to increase the other organs of the plant morphology rendering.

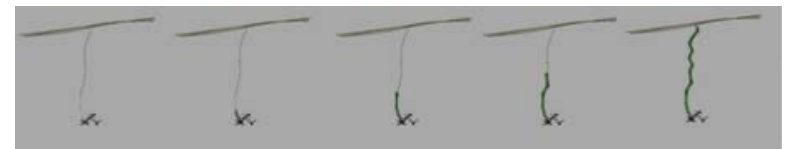

FIGURE VII. THE GROWTH PROCESS BY TWINING THE VINE BY HAND

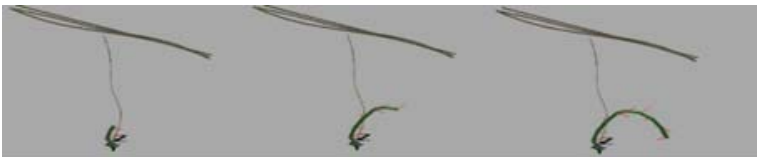

(a)

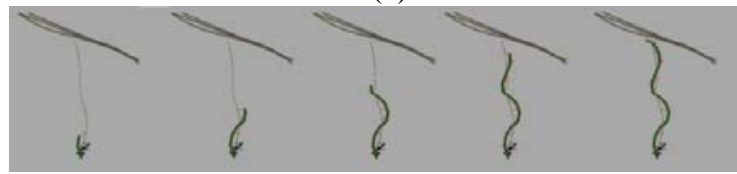

(b)

FIGURE VIII. THE GROWTH PROCESS BY TENDRILS EXPLORATION

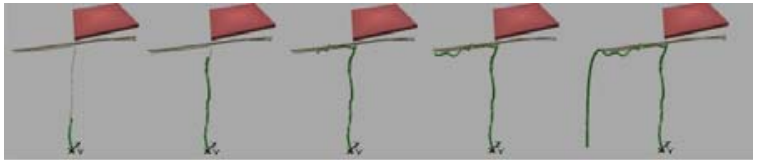

FIGURE IX. THE GROWTH PROCESS OF CUCUMBER IN GREENHOUSE

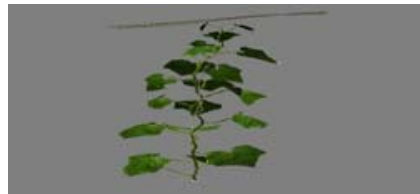

FIGURE X. THE COMPLETE FORM OF 3D CUCUMBER

\section{ACKNOWLEDGMENT}

This research war financially supported by the science foundation of Liaoning Province (L2014265) and (L2015484).

\section{REFERENCES}

[1] Chun jiang Zhao, Shenglian Lu, Xinyu Guo,etc. Discussion on Technical System and Digital Plant [J].Agricultural Sciences in China,2010, 43(10):2023-2030.

[2] Xiaoyuan Xu.Artificial Fish - Artificial Life for Computer Animation [M]. Beijing: Tsinghua University Press, 2001.

[3] K. Perlin, A. Goldberg. IMPROV: A System for Scripting Interactive Actors in Virtual Worlds[C]. Proc. of SIGGRAPH 96, pp. 205-216, New Orleans, LA, ACM SIGGRAPH, 1996. 
[4] N. I. Badler, C. B. Phillips and B. L. Webber. Simulating Humans: Computer Graphics, Animation, and Control [M]. Oxford Univ. Press, 1993.

[5] im Hanan, Przemyslaw Prusinkiewicz, Myron Zalucki, David Skirvin. Simulation of insect movement with respect to plant architecture and morphogenesis [J]. Computers and Electronics In Agriculture. 2002, 35(3), 225-269.

[6] Benes Bedrich and Enrique D. Espinosa, Modeling Virtual Ecosystems with the Proactive Guidance of Agents[J], IEEE Computer Animation and Social Agents, 2003.

[7] Zhongbin Su, Fanjiang Meng, Kang Li, Ping Zheng.Research and Exploration for Virtual Plant Model Based on Agent technology[J].Journal of Agricultural Engineering. 2005, 21 (8):114-117.

[8] Xiaoming Li, Chunjiang Zhao, Ping Zheng.The Plant Growth System Architecture based on Agent [J].Journal of Northeast Agricultural University.2010,41(8): 127-131.

[9] Lu Sheng lian, Zhao Chun jiang, Guo Xin yu, Li Chang-feng. BiLayered Mass-Spring Model for Leaf Motions [J]. Journal of System Simulation. 2009.14.

[10] Lu Sheng lian, Zhao Chun jiang, Guo Xin yu, Li Chang-feng. Efficient Simulating Interactive Deformation of Virtual Plant[C]. Third International Symposium on Plant Growth Modeling, Simulation, Visualization and Applications (PMA09), Beijing, China, November, 2009. pp. 395-398 